\title{
Abstracts Author Index by abstract number
}

A

Akhtar, S., 3

Ambrose, K., 16

B

Blechman, J.A., 5

Boyle, D., 17

D

De Sitter, L.L., 13

Dundas, C., 17

$\mathbf{E}$

Emerson, S., 16

F

Fernandes, R., 2

Fess, E.G., 2

Flemig, D., 4

Fox, A.F., 13

H

Hannifan, M., 5

Head, B.A., 9, 15

Heitner, R., 6

Hoit, F.V., 10

$\mathbf{K}$

Kennedy, W., 16

Kestenbaum, M.G., 10

L

Leonard, K., 3

Li, D., 2

Lindley, L.C., 10

Lynn, J., 13

M

Mackie, A., 5

Mauriello, C.J., 12

Mazzacco, M., 3

Merrifield, S., 17

Murphy, K., 17
$\mathbf{N}$

Nickels, K.S., 4

$\mathbf{P}$

Paxton, J.J., 13

Payne, C., 1

Pelly, N., 14

Perez, E., 11

Peters, B.A., 15

Pfeifer, M., 9

Pierson, C. 5

$\mathbf{R}$

Richar, C., 10

Rounds, L., 11

Rugh, D., 7

Runnels, S.A., 8

S

Sanders, J., 4

Schapmire, T., 9

Schroeder, B., 17

Schwoyer-Morgan, T.L., 12

Shah, G., 12

Skinner, K., 13

Smith, J., 1

Souza, J., 17

Srinivasan, V., 3

Steinhorn, D., 10

Stempek, M., 17

V

Vance, C., 8

VandeKieft, G., 17

W

Wegner, K., 17

Weeks, J.M., 14

Williamson, R., 13

Wilson, B., 13

Y

Youngs, K., 16 